\title{
Monitoring of the Changes in the Global Forest Cover Using Data Mining ${ }^{1}$
}

\author{
Vipin Kumar \\ William Norris Professor and Head, Department of Computer Science and Engineering, \\ University of Minnesota
}

\begin{abstract}
Assessing change in forest cover is of critical importance in studying natural and anthropogenic impacts on natural ecosystems. In particular, forest degradation accounts for almost $20 \%$ of anthropogenic greenhouse gas emissions (GHG) and thus is a significant driver of climate change, which, in turn can impact the health of the global ecosystem. Hence there has been a significant increase in international efforts such as the United Nations Program on Reducing Emissions from Deforestation and Forest Degradation (UN-REDD). In addition to politically negotiated treaties, a market based approach has been proposed in which corporations or countries that are significant emitters of atmospheric carbon offer monetary payments for forest preservation in exchange for carbon credits to be used in a carbon trading system.
\end{abstract}

A key ingredient for effective forest management, whether for carbon trading or other purposes, is quantifiable knowledge about changes in forest cover. Rich amounts of data from remotely sensed images are now becoming available for detecting changes in forests or more generally, land cover. However, in spite of the importance of this problem and the considerable advances made over the last few years in high-resolution satellite data acquisition, data mining, and online mapping tools and services, end users still lack practical tools to help them manage and transform this data into actionable knowledge of changes in forest ecosystems that can be used for decision making and policy planning purposes. Providing this actionable knowledge requires innovations in a number of technical areas: (i) identification of changes in global forest cover, (ii) characterization of those changes, and (iii) discovery of relationships between the number, magnitude, and type of these changes with natural and anthropogenic variables. To realize progress in the above areas, a number of computational challenges in spatio-temporal data mining need to be addressed. Specifically, analysis and discovery approaches need to be cognizant of climate and ecosystem data characteristics such as seasonality, inter-region variability, multi-scale nature, spatio-temporal autocorrelation, high dimensionality and massive data size. This talk describes our initial efforts, achievements and challenges in addressing some of the above areas.

\footnotetext{
${ }^{1}$ Research funded by Planetary Skin Institute, NSF, NASA, CISCO, and MN Futures Program.
} 
